\title{
Appoximationism as a new art form in Art Therapy.
}

Dr Bheemaiah, Anil Kumar, A.B Seattle W.A 98125

miyawaki@yopmail.com

\begin{abstract}
:
Approximationism as an art form is defined in a deep definition of human nature, as a model maker, in correlations and autocorrelations with varying degrees of naturalness, human cognition, is defined as symbolic dynamism and abstractionism, in a spectrum, with algorithms and data mining, defined as approximationism in visual declaration, symbolic mathematics, natural language and metaphors, an ontological API and Taxonomy in cognition. Various metrics of formal elements of approximationism for art therapy are the topic of a future publication.

Keywords: approximationism, ontology API, taxonomy, complexity theory, metrics, visual dynamism, visual declaration, graphics calculators, docking stations.

What:

Mathematics is a universal language, but Godel's incompleteness theorem proves it's incompleteness, not even closed form expressions for natural language or for consciousness are possible, so approximations are the solution, every model we make and data we collect are mere approximations. Is art too an approximation and a visual description of a model? This is approximationism, the art of approximations of life, art as an approximation.

We explore the use of approximation art in art therapy and data mining or sketch to coding, for the formal definitions and the programming of approximationist art therapy.
\end{abstract}

\section{Introduction.}

To quote Richard Feynman :

"I have approximate answers, and possible beliefs, and different degrees of certainty about different things, but I'm not absolutely sure of anything."

The mind creates models of the world of senses, immersed in this sensuality, one creates and persists in the written tongue, natural language, mathematics and code about the world. But these are mere approximations, metaphors and metaphysics. Thus flows the formal definition of approximationism, in visual form, as symbols, as state diagrams, as mathematics, or as images or symbolic dynamism.

As the mind makes correlations and autocorrelations, the basket of associations is much like data mining algorithms, thus algorithms are really one form of approximationism, an art therapy, so is data mining and the gathering of data. All mathematical modeling is part of this approximationism and can be part of art therapy. 


\section{Formal Elements:}

The formal elements of any art, of images or of dynamic visuals, both passive and interactive media, is of size, shape, form, color, line and texture. (Havens 2018; "The Formal Elements in Art" 2012)The formal elements have many emergent properties, approximation and incompleteness is one.

We describe the ontological form of the visual definition as an approximation to the infinite nature of naturalness, as being completely arbitrary in the complexity of the human cognition, increasing in sophistication, the more a person ruminates and of various degrees of accuracy in approximation. The definition of metrics of simplicity, minimalism and naturalness are a topic of a future publication.

\section{Coding:}

The coding can be in abstractionism or in impressionism or a spectrum of form, symbols, dynamism, in a range of mathematics and formal elements, of flow or interaction. Given emerging fields of computational art, art is easily defined in a ontological API and Taxonomy and the existence of iterative and recursive algorithms. Algorithmics is itself approximationism and so is data collection, modelling and visualization, an art in itself.

Functions, regression, distributions and model theory are all forms of approxionatism. The visual display of such functions, models and approximations by time series or closed form decompositions, rendered in visuals form either as plots, graphs or the symbols itself form approximationist art forms.
A programmable graphics calculator as the medium.

The calculator transcends the role as a tool of engineering and mathematics and in mathematical, symbolic and code based art, takes on the role of an artist's tool, with casting to dock stations, including lapdocks and wall dock stations, art is truly universal and even functional as wall dock stations display approximationism. Life itself is defined in the framework of calculators and dock stations.

Examples of approximationism by the author.

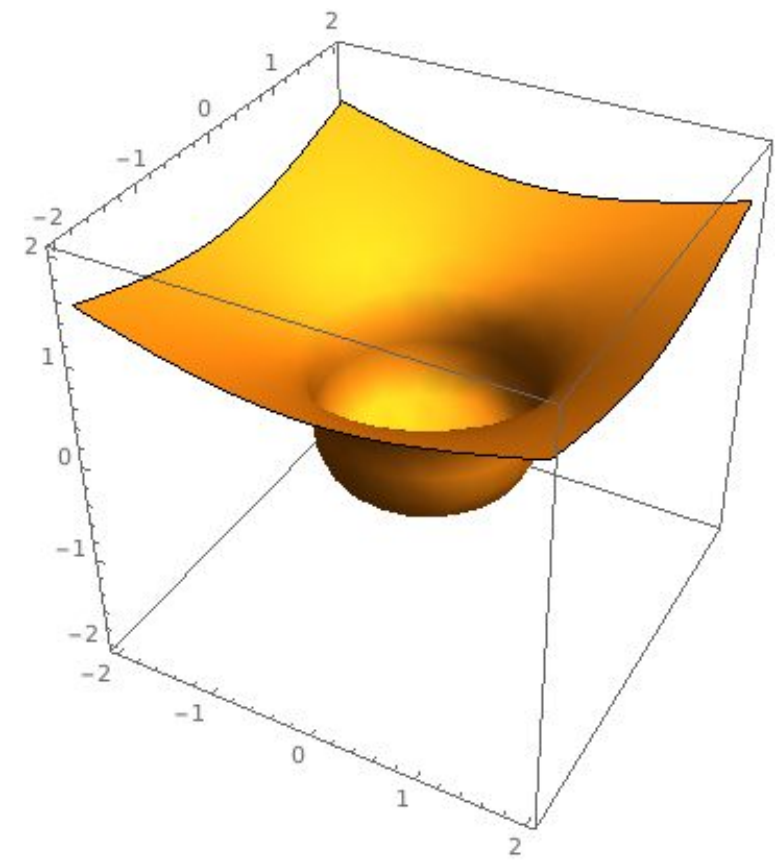

A contour with a single singular attractor, a simple energy landscape, sucking all to the base , a stability theorem. At they lie at the base, unable to climb the contour, they reflect on who they are, how they think.

Will they mind melt?("Contour Mathematica by Bheemaiaha on DeviantArt" n.d.) 


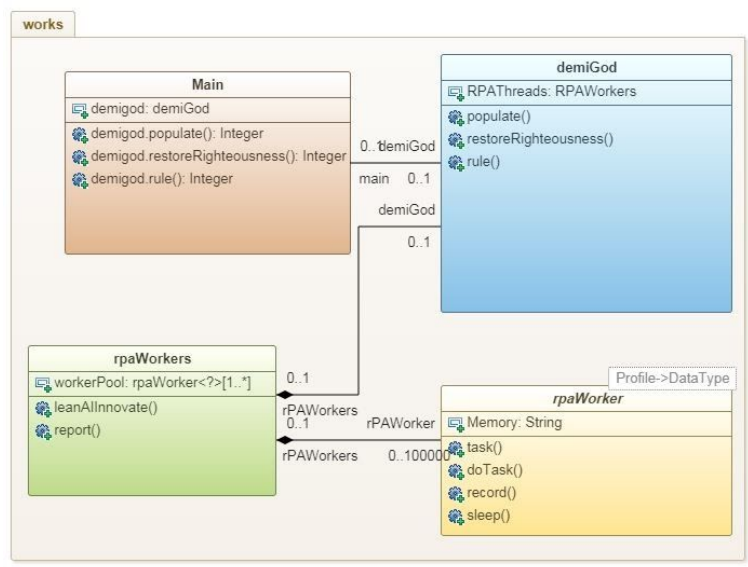

This UML is a design for a Cyber World of 100,000 RPA threads, they work and are ruled by a DemiGod, who makes sure there is righteousness. In this world of the Law Abiding Citizens, there is a songbird who sits on top of a pole and sings out the rules, You the modern day white collar worker have become nothing more than a R.P.A, with rules to automate you and colored tracks to follow as you lead your life from 9 am to $5 \mathrm{pm}$ and then are 'parked' at Home. ("Filter out the Misguided by Bheemaiaha on DeviantArt" n.d.. "Population Explosion by Bheemaiaha on DeviantArt" n.d.)

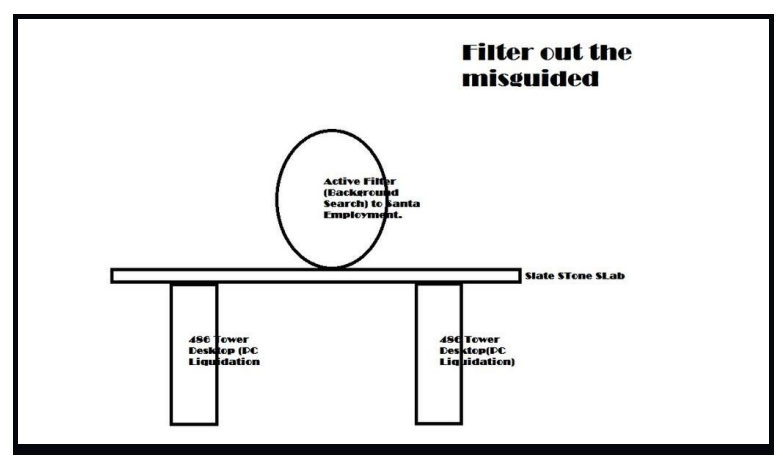

My mother once was ordered by a memo to buy school benches made in central prison, Bangalore. Today that prison is defunct and instead a beautiful park called Freedom Park stands there to commemorate all the freedom fighters and so called patriots mislabeled "terrorists" who were housed there. Freedom
Park is a sculpture garden with many sculptures, Anna Hazare a Gandhian also protested against corruption here.

My abstract digital schematic art is a bench, representing both the patriot and the pre-mature optimizer, a bench from two 486 tower desktops from PC liquidation representing sick industries with government liquidation, a symbol of bankruptcy, and a Egg, a metaphorical egg that filters the misguided youth out of this bench and advises them against violence and premature optimization, instead employing them with a kind Santa, during the holidays or with a time slip, through out the year making gifts for good children.("Filter out the Misguided by

Bheemaiaha on DeviantArt" n.d.)

\section{Discussion.}

In (Bheemaiah, n.d.), 2019, Dr Bheemaiah, talks of reactive stream based programming for cross and autocorrelation operators as approximations in models as perception and naturalness in it, thus reactive programming, as popularized by Netfix, (Havens 2018)itself becomes approximationism, and so do the basket of associations as a data mining interpretation of art and perception. Given formal methods in approximation, by procedural A.I, regression, statistical methods and deep learning, we have developed better and better metrics for quantifying the success of our models, the deep learning and the regressions, in describing life, Ast art Google spearheaded the dreamscapes of machine dreams, dreams in the neural networks and the convolution of images in a strange eerie juxtaposition, and a fluidic space time perception, all in a dreamy state reality. Art therapy stems from this source of machine dreams in broad definitions of symbolism, a symbolism central to circuit schematics and 
algebraic expressions and defined in geometries of nature. This approximationism flows from the very source of the Java LAnguage, from the very formal analysis of programming languages as more than visual declaration, but a form of approximationism, of breath in the programming and the coding, of the side effects and of life itself.

\section{Future Work.}

Future work is a critical look at minimalism, and sophistication in the natural data mining, as described in approximationism, like algorithmics and complexity theory, we define an analog to art formalism, and the models, the degree of approximation and its distance from minimalism and the simplicity of nature. And the degree of complexity of humans is quantified by metrics in future work.

Naturalness forms the basis of art and art therapy is naturally defined in a metric for this separation of man from nature of the naturalness of mankinds approximationism.

\section{References.}

Bheemaiah, Anil Kumar. n.d. "Correlation Transformational Operators in Reactive Streams." https://doi.org/10.31224/osf.io/5wz7d. "Contour Mathematica by Bheemaiaha on DeviantArt." n.d. Accessed June 8, 2020. https://www.deviantart.com/bheemaiaha/art /Contour-mathematica-745909950.

"Filter out the Misguided by Bheemaiaha on DeviantArt." n.d. Accessed June 8, 2020. https://www.deviantart.com/bheemaiaha/art /Filter-out-the-misguided-791341677.

Havens, Timothy. 2018. "Netflix." From Networks to Netflix. https://doi.org/10.4324/9781315658643-30.

"Population Explosion by Bheemaiaha on DeviantArt." n.d. Accessed June 8, 2020. https://www.deviantart.com/bheemaiaha/art
/Population-Explosion-776558670.

"The Formal Elements in Art." 2012.

HardleyArt. March 25, 2012.

https://hardleyart.wordpress.com/the-formal -elements-in-art/. 